\title{
Fermionic superfluidity: From high Tc superconductors to ultracold Fermi gases
}

\author{
Qijin Chen, Chih-Chun Chien, Yan He, and K. Levin \\ James Franck Institute and Department of Physics, University of Chicago, Chicago, Illinois 60637, USA
}

(Dated: October 24, 2018)

\begin{abstract}
We present a pairing fluctuation theory which self-consistently incorporates finite momentum pair excitations in the context of BCS-Bose-Einstein condensation (BEC) crossover, and we apply this theory to high $T_{c}$ superconductors and ultracold Fermi gases. There are strong similarities between Fermi gases in the unitary regime and high Tc superconductors. Here we address key issues of common interest, especially the pseudogap. In the Fermi gases we summarize recent experiments including various phase diagrams (with and without population imbalance), as well as evidence for a pseudogap in thermodynamic and other experiments.
\end{abstract}

Keywords: high Tc superconductor, ultracold Fermi gas, superfluidity, Feshbach resonance

Superfluidity in ultracold trapped fermionic gases has been one of the most exciting, rapidly evolving research subjects in the past few years. [1, 2, 3, 4, 5, 6]. It has captured the attention of both condensed matter and atomic physics community, as well as other fields such as nuclear and quark matter [7]. Atomic Fermi gases have strong similarities to superconductors. Breakthroughs in cooling techniques in recent years have made it possible to cool an atomic Fermi gas to quantum degeneracy temperature and thus form a superfluid. What is remarkable about the atomic Fermi gases is their tunability and controllability. Using a Feshbach resonance, one can tune the attractive two-body interaction from weak to strong, and thereby make a smooth crossover from a BCS type of superfluid to a Bose-Einstein condensation (BEC) [8]. BCS-BEC crossover has been of interest to the condensed matter community for a long time, and has been argued [9] to be relevant to high $T_{c}$ superconductivity.

Most of the theories that are proposed to explain high $T_{c}$ superconductivity are based on the pairing of electrons, as in BCS theory. While the pairing mechanism in the cuprate superconductors is far from being clear, there is no ambiguity about the pairing interaction in atomic Fermi gases. The attractive pairing potential between the fermionic atoms of different spins has been well characterized and can be computed from first principle quantum mechanics calculations. Here we presume that cuprate superconductivity originates from electron pairing, so that studying superfluidity in atomic Fermi gases may provide insights into the high $T_{c}$ superconductivity or vice versa $[9,10,11]$.

Importantly, preformed pairs are an inevitable consequence as the pairing interaction increases. At the unitary scattering limit, the superfluid transition temperature $T_{c}$ is very high in units of the noninteracting Fermi energy $E_{F}$. The atomic Fermi gases often have a relatively higher transition temperature than the cuprates. For the latter $T_{c} \lesssim 0.1 E_{F}$ is somewhat lower, owing in part to their low dimensionality. Except in the extreme BCS limit, preformed pairs contribute a fermionic excitation gap, i.e., a pseudogap, even in the normal state. Such a pseudogap is common to both high $T_{c}$ superconductors and atomic Fermi gases.

In the absence of a population imbalance, superfluidity is associated with a gas of fermions with dispersion $\xi_{\mathbf{k}}=\epsilon_{\mathbf{k}}-$ $\mu$, subjected to an attractive interaction between two different spin states, $U_{\mathbf{k}, \mathbf{k}^{\prime}}=U \varphi_{\mathbf{k}} \varphi_{\mathbf{k}^{\prime}}$, where $U<0$ is the coupling strength. For the cuprates, $\epsilon_{\mathbf{k}}=2 t_{\|}\left(2-\cos k_{x}-\cos k_{y}\right)$, and for atomic Fermi gases $\epsilon_{\mathbf{k}}=k^{2} / 2 m ; \mu$ is the fermionic chemical potential, $t_{\|}$is the in-plane hopping integral, and we have set the lattice constant to 1 . Here and throughout, we assume natural units and set $\hbar=k_{B}=c=1$. The function $\varphi_{\mathbf{k}}$ reflects the pairing symmetry. For the cuprates, $\varphi_{\mathbf{k}}=$ $\cos k_{x}-\cos k_{y}$; for the Fermi gases, the interaction is shortranged, and taken to be a contact potential so that $\varphi_{\mathbf{k}}=1$.

The Hamiltonian is given by

$$
\begin{aligned}
H- & \mu N=\sum_{\mathbf{k} \sigma} \xi_{\mathbf{k}} a_{\mathbf{k} \sigma}^{\dagger} a_{\mathbf{k} \sigma} \\
& +\sum_{\mathbf{k k}^{\prime} \mathbf{q}} U_{\mathbf{k}, \mathbf{k}^{\prime}} a_{\mathbf{k}+\mathbf{q} / 2 \uparrow}^{\dagger} a_{-\mathbf{k}+\mathbf{q} / 2 \downarrow}^{\dagger} a_{-\mathbf{k}^{\prime}+\mathbf{q} / 2 \downarrow} a_{\mathbf{k}^{\prime}+\mathbf{q} / 2 \uparrow},
\end{aligned}
$$

where $a^{\dagger}(a)$ is the creation (annihilation) operator of the fermions. This picture is often referred to as a one-channel model. The physics of a Feshbach resonance can be described by a two-channel model, which includes an open-channel, as in the one-channel model, and a closed-channel, which represents the effects of di-atomic molecules of a different total spin. The two-channel Hamiltonian is given by

$$
\begin{aligned}
H & -\mu N=\sum_{\mathbf{k}, \sigma} \xi_{\mathbf{k}} a_{\mathbf{k}, \sigma}^{\dagger} a_{\mathbf{k}, \sigma}+\sum_{\mathbf{q}}\left(\epsilon_{\mathbf{q}}+\nu-2 \mu\right) b_{\mathbf{q}}^{\dagger} b_{\mathbf{q}} \\
& +\sum_{\mathbf{q}, \mathbf{k}, \mathbf{k}^{\prime}} U\left(\mathbf{k}, \mathbf{k}^{\prime}\right) a_{\mathbf{q} / 2+\mathbf{k}, \uparrow}^{\dagger} a_{\mathbf{q} / 2-\mathbf{k}, \downarrow}^{\dagger} a_{\mathbf{q} / 2-\mathbf{k}^{\prime}, \downarrow} a_{\mathbf{q} / 2+\mathbf{k}^{\prime}, \uparrow} \\
& +\sum_{\mathbf{q}, \mathbf{k}}\left(g(\mathbf{k}) b_{\mathbf{q}}^{\dagger} a_{\mathbf{q} / 2-\mathbf{k}, \downarrow} a_{\mathbf{q} / 2+\mathbf{k}, \uparrow}+\text { h.c. }\right)
\end{aligned}
$$

where $b^{\dagger}(b)$ is the creation (annihilation) operator for the closed-channel molecules, whose dispersion is given by $\epsilon_{\mathbf{q}}=$ $q^{2} / 2 M$ with $M=2 m$. Here $\nu$ represents the magnetic detuning, which is used to tune the relative energy level splitting between the open- and closed-channels and thus change the overall effective interaction; the interaction increases as the field decreases. The last line in Eq. (2) represents the coupling between the two channels, with $g(\mathbf{k})=g \varphi_{\mathbf{k}}$.

Current studies of atomic Fermi gases have concentrated on ${ }^{6} \mathrm{Li}$ and ${ }^{40} \mathrm{~K}$. For both gases, the Feshbach resonances 
which are used to enhance the pairing interaction have a very big width. As a consequence, the closed-channel fraction becomes negligible [6, 12] even though the closed channel makes a big contribution to the effective pairing interaction. For example, for ${ }^{6} \mathrm{Li}$, both experiments [6] and our theoretical calculations [12] have demonstrated that the closed-channel population is extremely small in the unitary regime (of the order of $10^{-5}-10^{-4}$ ) and remains small in the entire range of magnetic field (i.e., pairing strength) which is accessed experimentally.

The net effect of this tiny closed-channel fraction is that, for many purposes, one can use the one-channel model, Eq. (1), provided one replaces $U$ with the overall effective interaction, $U_{e f f}(Q) \equiv U+g^{2} D_{0}(Q)$, where $D_{0}(Q) \equiv 1 /\left[i \Omega_{n}-\epsilon_{\mathbf{q}}^{m b}-\right.$ $\nu+2 \mu]$ is the bare propagator of the closed-channel bosons. Experimentally, the pairing interaction for atomic Fermi gases is usually parametrized by the dimensionless product, $1 / k_{F} a$, where $a$ is the $s$-wave inter-fermion scattering length, and $k_{F}$ is the Fermi wavevector defined in the noninteracting limit at $T=0$, satisfying $E_{F}=k_{F}^{2} / 2 m$.

In this way both high $T_{c}$ superconductors and atomic Fermi gases can be described by the same Hamiltonian but with different pairing symmetries and free-particle dispersions. The existence of a big pseudogap and the short coherence length in the cuprates suggest that they are in the intermediate regime between BCS and BEC [9]. This is often referred to as the "strongly interacting regime" in atomic Fermi gases, and is the most complex and interesting regime as well.

Our pairing fluctuation theory uses a $T$-matrix formalism. Instead of providing a detailed derivation, which can be found in Refs. [10, 11], here we recapitulate the key ingredients and observations of this formalism. Pairs consist of fermions which glue together via the attractive interaction, and the pair propagator represents a summation of multiple particle-particle scattering processes. Fermions acquire selfenergy via creation and destruction of a pair and a hole. In BCS theory, such pairs would be just the Cooper pairs in the condensate, and they contribute a self-energy $\Sigma_{s c}(K)=$ $-\Delta_{s c}^{2} G_{0}(-K) \varphi_{\mathbf{k}}^{2}$, where $\Delta_{s c}$ is the order parameter and $G_{0}(K)$ is the bare fermion Green's function. [Throughout, we use a four-vector notation as in Ref. [10], $K \equiv\left(\mathbf{k}, i \omega_{n}\right)$, $\sum_{K}=T \sum_{n} \sum_{\mathbf{k}}$, etc., and $\omega_{n}$ is the Matsubara frequency.] In general, finite momentum pairs contribute to the fermion self-energy via $\Sigma_{p g}(K)=\sum_{Q} t_{p g}(Q) G_{0}(Q-K) \varphi_{\mathbf{k}}^{2}$, where $t_{p g}(Q)=1 /\left[1+U_{e f f}(Q) \chi(Q)\right]$ is the $T$-matrix (pair propagator), with $\chi(Q)=\sum_{K} G_{0}(Q-K) G(K)$ being the pair susceptibility and $G(K)$ the full fermion Green's function. Below $T_{c}, t_{p g}(Q)$ diverges at $Q=0$ so that we can approximate $G_{0}(Q-K) \approx G_{0}(-K)$ in $\Sigma_{p g}$. As a result, we obtain $\Sigma_{p g}(K) \approx-\Delta_{p g}^{2} G_{0}(-K) \varphi_{\mathbf{k}}^{2}$, with

$$
\Delta_{p g}^{2} \equiv-\sum_{Q} t_{p g}(Q) .
$$

Here the key observation is that, under a reasonable approximation, the self-energy of the finite momentum pairs has the same form as $\Sigma_{s c}(K)$. Since these finite momentum pairs do not have phase coherence, their contributions lead to a pseudogap in the fermion dispersion. The total excitation gap $\Delta$ is given by

$$
\Delta^{2}=\Delta_{s c}^{2}+\Delta_{p g}^{2}
$$

and the fermionic quasiparticle dispersion becomes $E_{\mathbf{k}}=$ $\sqrt{\xi_{\mathbf{k}}^{2}+\Delta^{2} \varphi_{\mathbf{k}}^{2}}$. The fact that the gaps add in quadrature can be understood from the observation that $\Delta_{s c}^{2}$ and $\Delta_{p g}^{2}$ are proportional to the density of the condensed and noncondensed pairs, respectively.

The effective dispersion $\Omega_{\mathbf{q}}$ and chemical potential $\mu_{\text {pair }}$ can be determined by a Taylor expansion of $t_{p g}^{-1}$, which, after analytical continuation $\left(i \Omega_{n} \rightarrow \Omega+i 0^{+}\right)$, can be written as

$$
t_{p g}^{-1}=Z\left(\Omega-\Omega_{\mathbf{q}}+\mu_{\text {pair }}\right),
$$

where $Z$ is the inverse residue [11], and we have neglected the small imaginary part [11]. The pairing instability condition, or equivalently the BEC condition for pairs, requires

$$
t_{p g}^{-1}(0)=0
$$

so that we have $\mu_{\text {pair }}=0$ below $T_{c}$. One can readily see that $\Delta_{p g} \rightarrow 0$ as $T \rightarrow 0$. Therefore, this finite $T$ theory is consistent with the BCS-Leggett ground state [8], which has been widely assumed in the literature.

The presence of the pseudogap necessarily suppresses the superfluid transition $T_{c}$. This can be seen from the relationship between $\Delta$ and $T$, as determined by a mean-field BCS gap equation: $T$ decreases as $\Delta$ increases, and $T_{c}$ simply corresponds to the lowest $T$ at which $\Delta=\Delta_{p g}$.

Our theory can be summarized in three self-consistent equations: the gap equation (6), the pseudogap equation (3), and the number equation

$$
n=2 \sum_{K} G(K) .
$$

They can be used to solve for $\Delta, \Delta_{p g}, \Delta_{s c}, \mu$ and $T_{c}$ as a function of $U$ (or $U_{\text {eff }}$ ) or $1 / k_{F} a$ and $T$.

The present theory naturally leads to the following important observations. (i) The fundamental statistical entities in these superfluids are fermions. We measure the "bosonic" (or pair) degrees of freedom indirectly via the fermionic gap parameter $\Delta(T)$. (ii) As we go from BCS to BEC, pairs will begin to form at a temperature $T^{*}$ above $T_{c}$. This pair formation is associated with a normal state pseudogap. (iii) In general there will be two types of excitations in these BCS$\mathrm{BEC}$ crossover systems. Importantly in the intermediate case the excitations consist of a mix of both fermions and bosons.

It should be pointed out that effects of pairing fluctuations were first considered by Noziéres and Schmitt-Rink [13]. However, they only included them in the particle number equation; the absence of these effects in the gap equations necessarily leads to internal inconsistencies, say, in computing the superfluid density. In contrast to the present approach, the NSR scheme also predicts that $T_{c}$ approaches its BEC asymptote from above for an $s$-wave superfluid in three-dimensional (3D) continuum space. The NSR treatment, due to its relative simplicity, has been used by others in the recent literature [14, 15]. 


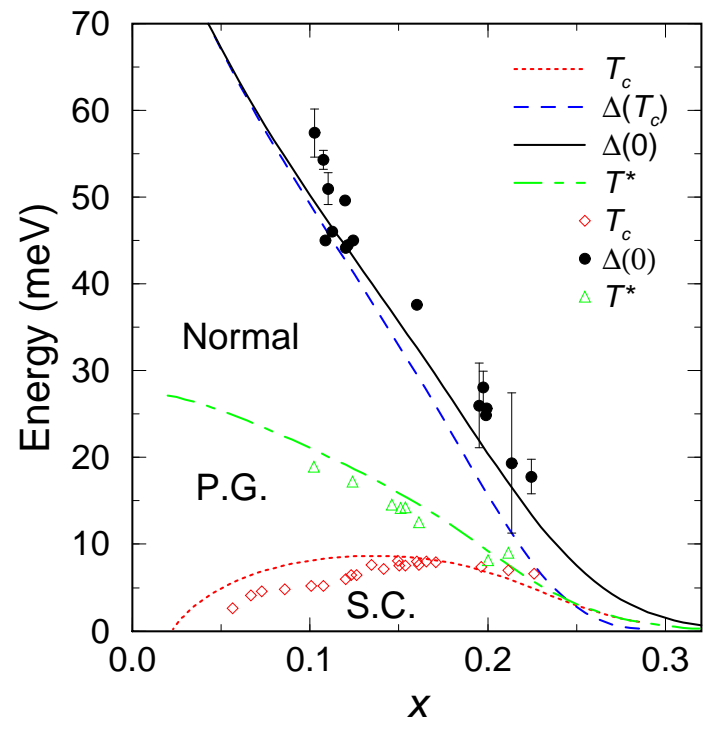

Figure 1: Cuprate phase diagram showing $\Delta(0), T_{c}, \Delta_{p g}\left(T_{c}\right)$, and $T^{*}$, calculated for $-U / 4 t_{0}=0.047$, and $t_{\perp} / t_{\|}=0.003$. Shown as symbols are experimental data. The normal, pseudogap, and superconducting phases are labeled with "Normal", "P.G.", and "S.C.", respectively. From Ref. [16].

A self-consistent treatment of finite-momentum pair excitations is crucial. This is more so in atomic Fermi gases, where one cannot directly measure the temperature except in the extreme BCS and BEC limits. Quantitatively reasonable knowledge of temperature effects is necessary in order to determine temperature and related physical properties in the BCS-BEC crossover regime.

We first apply our pairing fluctuation theory to high $T_{c}$ superconductors. In Fig. 1 we plot the calculated phase diagram for $\mathrm{Bi}_{2} \mathrm{Sr}_{2} \mathrm{CaCu}_{2} \mathrm{O}_{8+\delta}$ (Bi2212) and $\mathrm{YBa}_{2} \mathrm{Cu}_{3} \mathrm{O}_{7-\delta}$ (YBCO) single crystals, as well as present a comparison with experimental data. The horizontal axis is the hole doping concentration per unit cell, $x=1-n$. Here we show theoretical calculations and experimental data for zero $T$ excitation gap $\Delta(0)$, superconducting transition temperature $T_{c}$, and pairing onset temperature $T^{*}$. We also show the theoretical result for $\Delta\left(T_{c}\right)$, the pseudogap at $T_{c}$.

To obtain this phase diagram, we fit with one parameter $-U / 4 t_{0}$ such that it gives the right value of $T_{c}$ at optimal doping around $x=0.15$. Here $t_{0}$ is the bare in-plane electron hopping matrix element, in the absence of the Coulomb repulsion. It is related to $t_{\|}$via $t_{\|} \approx t_{0} x$. One would obtain essentially the same phase diagram, if one parametrized the variable attractive interaction by simply fitting $T^{*}(x)$ to experiment. Thus, the underdoped regime sees effectively a stronger pairing interaction, $-U / 4 t_{\|}$. It is evident that our simple model gives a (semi-)quantitatively satisfying phase diagram, in agreement with experiment.

It should be noted that, in contrast to the $s$-wave, 3D Fermi gas, the $d$-wave symmetry and the lattice periodicity in the cuprates makes $T_{c}$ vanish before the BEC regime can be reached as the couping strength increases [17]. This intro-
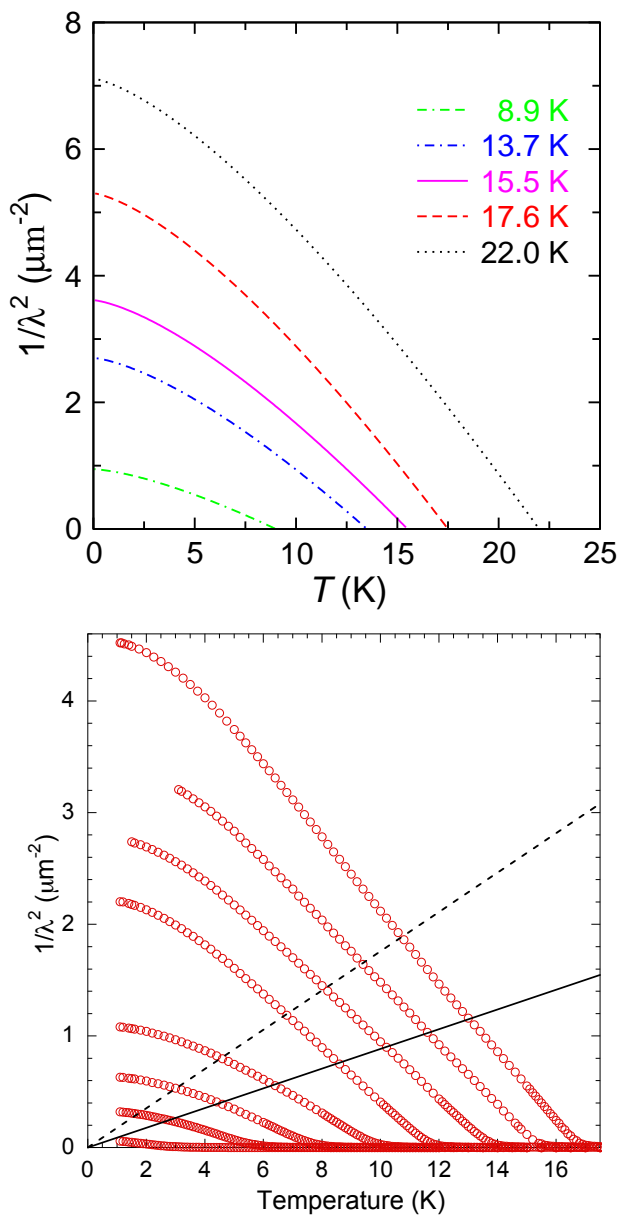

Figure 2: Comparison of superfluid density between theory and experiment. Top panel: Theoretical calculation for underdoped cuprate superconductors; Bottom panel: Experimental data from Ref. [18] in the extreme underdoped regime of YBCO.

duces a lower critical doping $x_{c}$ in the phase diagram shown in Fig.1.

Other applications of BCS-BEC crossover theory to the cuprate superconductors include studies [10] of the quasiuniversal behavior of the normalized superfluid density $n_{s}(T) / n_{s}(0)$ as a function of $T / T_{c}$ for various doping concentrations. Experimental data from Ref. [18] are plotted in the lower panel of Fig. 2 for a series of very underdoped cuprates. The universality apparent in these data presents a puzzle for conventional theories of the penetration depth which invoke only fermionic excitations of the condensate. This is because the fermionic contributions are expected to reflect the strong variations in the excitation gap $\Delta(x)$ associated with the different hole concentrations $x$, thereby leading to highly non-universal behavior. In the theory plot of the upper panel (Figure 2) bosonic or pair excitations contribute an extra mechanism for destroying superfluidity. They compensate for the decrease of the quasiparticle contribution $\left(\propto T_{c}(x) / \Delta(x)\right)$ as the system evolves from overdoping to underdoping. As a result, the slope of the superfluid density remains nearly doping independent, with only a small system- 


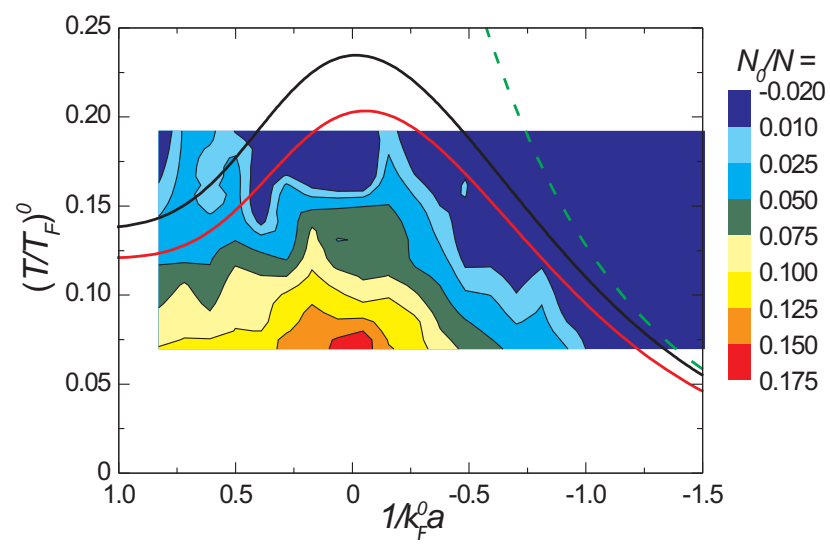

Figure 3: (color) Phase diagram of ${ }^{40} \mathrm{~K}$. A contour plot of the measured condensate fraction $N_{0} / N$ as a function of $1 / k_{F}^{0} a$ and effective temperature $\left(T / T_{F}\right)^{0}$ is compared with theoretically calculated contour lines at $N_{s} / N=0$ (black curve) and 0.01 (red curve). The overall trend of the experimental contour of $N_{0} / N=0.01$ and the theoretical line for $N_{s} / N=0.01$ are in good agreement. The dashed line represents the naive BCS result, $T_{c} / T_{F}^{0} \approx 0.615 e^{\pi / 2 k_{F}^{0} a}$. From Ref. [21].

atic variation with $x$. The comparison between theory and recent experiments from the UBC group [18] shows quite good agreement. Recently, the bosonic nature of the superfluid density in underdoped cuprates has also been recognized by other groups [19, 20].

We turn now to experiments in cold atom systems. Here we [11, 22] need to include the trap potential $V(r)$. To this end, we use a local density approximation (LDA) by replacing $\mu \rightarrow \mu(r) \equiv \mu-V(r)$. Here $\mu \equiv \mu(0)$ is the global chemical potential and $V(r)=m \bar{\omega}^{2} r^{2} / 2$ for a harmonic trap with mean angular frequency $\bar{\omega}$. The number constraint enters for the entire trap, $N=\int \mathrm{d}^{3} r n(r)$. Below $T_{c}$, there exists a superfluid core within $r<R_{s c}$. For $r>R_{s c}, \mu_{\text {pair }}<0$, and the gap equation has to be modified to reflect this non-zero $\mu_{\text {pair }}: t_{p g}^{-1}(0)=Z \mu_{\text {pair }}$.

Before we compare with experiment, we have to know the temperature of the system. Experimentally, temperature is measured at the BCS [1] or BEC end [23], before or after the experiment is performed in the strongly interacting regime. This BCS or BEC state is adiabatically connected to the state where experiments are done via a slow magnetic field sweep. By including self-consistently finite momentum pair excitations, our pairing fluctuation theory enables us to calculate the entropy $S(T)$ at an arbitrary magnetic field. Then we can map the physical temperature $T$ onto that measured in the BCS or BEC limit, $T^{0}$, or vice versa [24].

In Fig. 3. we show the phase diagram for ${ }^{40} \mathrm{~K}$ and compare our calculations with experimental data [21]. Here all temperatures are measured in (or converted into) the free Fermi gas limit. In addition, $T_{F}^{0} \equiv k_{F}^{0} / 2 m$ is the Fermi temperature at the trap center in the noninteracting limit. The experimental superfluid phase boundary is given by the $1 \%$ condensate fraction contour line; it is hard to locate experimentally where the condensate vanishes precisely. This should be compared with

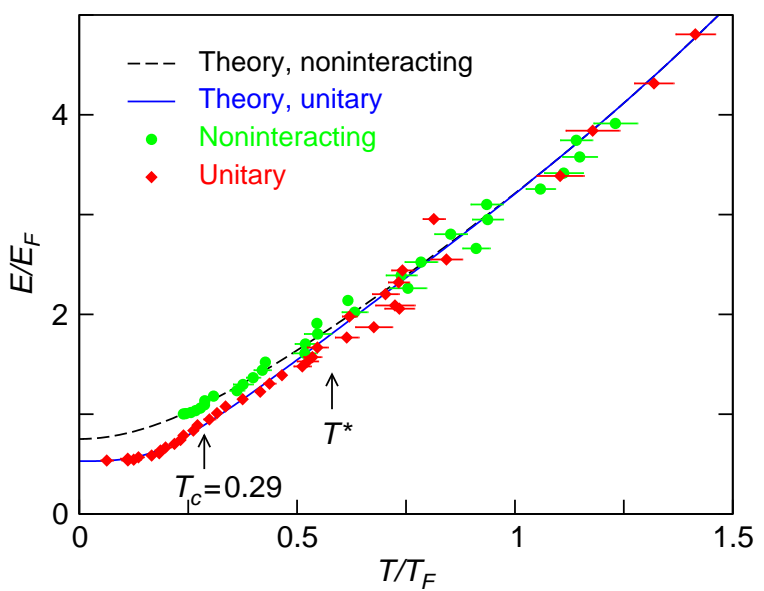

Figure 4: (color) Energy per particle as a function of $T$ for ${ }^{6} \mathrm{Li}$ at unitarity. The fact that the experimental data (symbols) (and the two theoretical curves) for noninteracting and unitary Fermi gases do not merge until higher $T^{*}>T_{c}$ is consistent with the presence of a normal state pseudogap. From Ref. [25].

the (red) theory curve. Here we [21] identify the condensate fraction with the superfluid density. The agreement between experiment and theory is reasonably good. It should be noted that both experiment and theory show a maximum around unitarity, $1 / k_{F}^{0} a=0$, which has also been observed in ${ }^{6} \mathrm{Li}$ phase diagrams [3].

We next turn to a detailed comparison of theory and experiment for thermodynamics. Figure 4 presents a plot of energy $E$ as a function of $T$ comparing the unitary and noninteracting regimes for ${ }^{6} \mathrm{Li}$. The solid curves are theoretical while the symbols are experimental data [25]. A realistic Gaussian trap with the experimentally given trap depth was used. There has been a recalibration of the experimental temperature scale [25] in order to plot theory and experiment in the same figure. The latter was determined via Thomas-Fermi fits to the density profiles [22]. To arrive at the calibration, we applied the same fits to the theoretically produced density profiles.

Good agreement between theory and experiment is apparent in Fig. 4 It should be emphasized that there is no fitting parameter in our calculations. The temperature dependence of $E$ reflects primarily fermionic excitations at the edge of the trap [24], although there is a small bosonic contribution as well. Importantly one can see the effect of a pseudogap in the unitary case. That is, the temperature $T^{*}$ is visible from the plots as that at which the non-interacting and unitary curves merge. This corresponds roughly to $T^{*} \approx 2 T_{c}$. Evidence of a pseudogap at unitarity has also been observed more directly in a radio-frequency excitation gap experiment [23].

Finally, we turn to the effects of population imbalance, which have recently been one of the hottest subjects in atomic Fermi gases. We consider both the homogeneous system [26], as well as trapped case, which can be found in Refs. [27, 28]. We define $n=n_{\uparrow}+n_{\downarrow}, \delta n=n_{\uparrow}-n_{\downarrow}$, and the polarization $p=\delta n / n$. There will be two number equations associated with the two spin species. The polarized superfluid phase is 


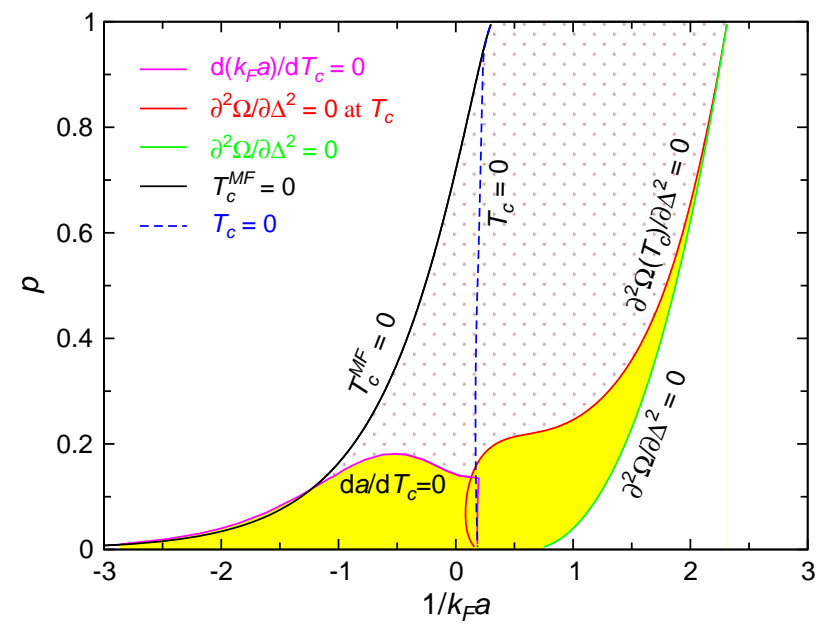

Figure 5: (color) Phase diagram in the $p-1 / k_{F} a$ plane, showing where intermediate temperature superfluidity associated with the Sarma phase (shaded region) exists. The (red) curve labeled by $\partial^{2} \Omega\left(T_{c}\right) / \partial \Delta^{2}=0$ is determined at corresponding $T_{c}$. The line defined by $\mathrm{a} / \mathrm{T}_{c}=0$ is given by the turning points $\left(p, 1 / k_{F} a\right)$ where $1 / k_{F} a$ reaches a local extremum as a function of $T_{c}$. At $T=0$, the entire region between the $T_{c}^{M F}=0$ and the $\partial^{2} \Omega / \partial \Delta^{2}=0$ lines is unstable against phase separation. However, a A stable polarized, intermediate temperature superfluid phase exists for the (yellow) shaded region. Homogeneous superfluids in the dotted region are unstable at any temperature. From Ref. [31].

conventionally called the "Sarma" phase [29], although here it is generalized away from the BCS limit originally considered by Sarma. Details are given in Ref. [26].

In Fig. 5, we present the zero $T$ phase diagram for a population imbalanced homogeneous Fermi gas in the $p-1 / k_{F} a$ plane. Here $T_{c}^{M F}$ is the solution of $T_{c}$ in a strict mean-field treatment. $\Omega$ is the thermodynamic potential. A superfluid solution is stable only if $\partial^{2} \Omega / \partial \Delta^{2}>0$. From this figure, we see that at any $p \neq 0$, there is a minimum threshold for $1 / k_{F} a$ (determined by $T_{c}^{M F}=0$ ) in order to have a superfluid solution. The point at which the solution becomes stable (determined by $\partial^{2} \Omega / \partial \Delta^{2}=0$ ) occurs in BEC regime. The (blue) dashed line indicates where $T_{c}$ vanishes. The dotted region (as well as the shaded region) in the figure indicates an unstable (Sarma) superfluid phase at $T=0$. The stable phases in this region are presumed to be either phase separated (PS) or possibly a Fulde-Ferrell-Larkin-Ovchinnikov state [30].

In addition to the zero $T$ phases, we also show in Fig. 5 where the system is stable at $T_{c}$. A stable finite temperature polarized superfluid exists in the (yellow) shaded region. This is a very interesting prediction of our theory which ap- pears consistent with experiment. More details can be found in Ref. [26].

Finally, we show in Fig. 6 the phase diagram at unitarity for a population-imbalanced Fermi gas in a harmonic trap with angular frequency $\omega$. Here $1 / k_{F} a=0$. Phase separation (labeled PS) occupies the lower $T$ portion of the phase diagram, where the gap $\Delta$ jumps abruptly to zero at some trap radius. At intermediate $T$, there is a (yellow-shaded) Sarma phase, where $\Delta$ vanishes continuously within the trap. It evolves into a (dotted region) pseudogap (PG) phase as the superfluid core vanishes at higher $T$. A normal $(\mathrm{N})$ phase without pairing always exists at even higher $T$. This phase diagram appears to be consistent with current experiments. One can easily see that the physics associated with population imbalance is much richer than in the absence of imbalance.

In conclusion, in this paper we have addressed commonalities particularly associated with the phase diagrams of ultracold trapped fermionic gases and high $T_{c}$ superconductors. These common features revolve around the scenario [9, 11] that the cuprates are somewhere intermediate between BCS and BEC. Importantly, this scenario has been directly realized in trapped Fermi gases. Here one sees considerable evidence for pre-formed pairs and the related fermionic pseudogap which appear reminiscent of their cuprate counterparts.

This work was supported by NSF PHY-0555325 and NSFMRSEC Grant No. DMR-0213745.

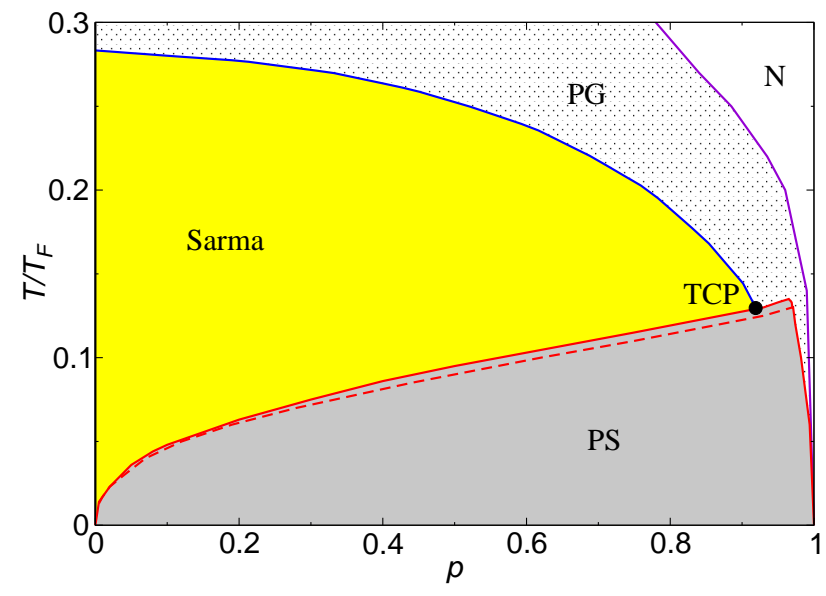

Figure 6: (color) Phase diagram of a population-imbalanced Fermi gas in a harmonic trap at unitarity. The solid lines separate different phases. Above the (red) dashed line but within the PS phase, the superfluid core does not touch the domain wall. Here "PG" indicates the pseudogapped normal phase. The black dot labeled "TCP" indicates the tricritical point.
[1] M. Greiner, C. A. Regal, and D. S. Jin, Nature (London) 426, 537 (2003).

[2] S. Jochim et al., Science 302, 2101 (2003).

[3] M. W. Zwierlein et al., Phys. Rev. Lett. 92, 120403 (2004).

[4] K. M. O'Hara, S. L. Hemmer, M. E. Gehm, S. R. Granade, and
J. E. Thomas, Science 298, 2179 (2002).

[5] T. Bourdel et al., Phys. Rev. Lett. 93, 050401 (2004).

[6] G. B. Partridge et al., Phys. Rev. Lett. 95, 020404 (2005).

[7] E. Gubankova, A. Schmitt, and F. Wilczek, Phys. Rev. B 74, 064505 (2006). 
[8] A. J. Leggett, in Modern Trends in the Theory of Condensed Matter, edited by A. Pekalski and J. Przystawa (SpringerVerlag, Berlin, 1980), pp. 13-27.

[9] A. J. Leggett, Nature Physics 2, 134 (2006).

[10] Q. J. Chen, I. Kosztin, B. Jankó, and K. Levin, Phys. Rev. Lett. 81, 4708 (1998).

[11] Q. J. Chen, J. Stajic, S. N. Tan, and K. Levin, Phys. Rep. 412 (2005) 1; Q. J. Chen, J. Stajic, and K. Levin, Low Temp. Phys. 32 (2006) 406.

[12] Q. J. Chen and K. Levin, Phys. Rev. Lett. 95, 260406 (2005).

[13] P. Nozières and S. Schmitt-Rink, J. Low Temp. Phys. 59, 195 (1985).

[14] M. Holland et al., Phys. Rev. Lett. 87, 120406 (2001).

[15] M. M. Parish, F. M. Marchetti, A. Lamacraft, and B. D. Simons, e-print cond-mat/0605744.

[16] Q. J. Chen, Ph.D. thesis, University of Chicago (2000), (unpublished).

[17] Q. J. Chen, I. Kosztin, B. Jankó, and K. Levin, Phys. Rev. B 59, 7083 (1999).

[18] D. M. Broun, P. J. Turner, W. A. Huttema, S. Ozcan, B. Morgan, R. Liang, W. N. Hardy, and D. A. Bonn, e-print cond-mat/0509223

[19] I. F. Herbut, Phys. Rev. Lett. 94, 237001 (2005).
[20] Y. J. Uemura, Physica B 374, 1 (2006).

[21] Q. J. Chen, C. A. Regal, M. Greiner, D. S. Jin, and K. Levin, Phy. Rev. A 73, 041601(R) (2006).

[22] J. Stajic, Q. J. Chen, and K. Levin, Phys. Rev. Lett. 94, 060401 (2005).

[23] C. Chin et al., Science 305, 1128 (2004).

[24] Q. J. Chen, J. Stajic, and K. Levin, Phys. Rev. Lett. 95, 260405 (2005).

[25] J. Kinast, A. Turlapov, J. E. Thomas, Q. J. Chen, J. Stajic, and K. Levin, Science 307 (2005) 1296.

[26] C.-C. Chien, Q. J. Chen, Y. He, and K. Levin, Phys. Rev. Lett. 97, 090402 (2006).

[27] C.-C. Chien, Q. J. Chen, Y. He, and K. Levin, Phys. Rev. A 74, 021602(R) (2006).

[28] C.-C. Chien, Q. J. Chen, Y. He, and K. Levin, e-print cond-mat/0612103

[29] G. Sarma, J. Phys. Chem. Solids 24, 1029 (1963).

[30] Y. He, C.-C. Chien, Q. J. Chen, and K. Levin, e-print cond-mat/0610274, to appear in Phys. Rev. A as a Rapid Communication.

[31] Q. J. Chen, Y. He, C.-C. Chien, and K. Levin, Phys. Rev. A 74, 063603 (2006). 